\title{
Tumour necrosis factor blockade for the treatment of erosive osteoarthritis of the interphalangeal finger joints: a double blind, randomised trial on structure modification
}

\author{
Gust Verbruggen, Ruth Wittoek, Bert Vander Cruyssen, Dirk Elewaut
}

Rheumatology Department Ghent University Hospital, Ghent, Belgium

\section{Correspondence to}

Gust Verbruggen, Ghent University Hospital,

Rheumatology Department De Pintelaan 185, BE 9000 Ghent, Belgium:

gust.verbruggen@ugent.be

GV and RW contributed equally to the study.

Received 10 January 2011 Accepted 6 October 2011 Published Online First 29 November 2011

\begin{abstract}
Background Adalimumab blocks the action of tumor necrosis factor- $\alpha$ and reduces disease progression in rheumatoid arthritis and psoriatic arthritis. The effects of adalimumab in controlling progression of structural damage in erosive hand osteoarthritis $(\mathrm{HOA})$ were assessed.
\end{abstract}

Methods Sixty patients with erosive HOA on radiology received $40 \mathrm{mg}$ adalimumab or placebo subcutaneously every two weeks during a 12-month randomized doubleblind trial. Response was defined as the reduction in progression of structural damage according to the categorical anatomic phase scoring system. Furthermore, subchondral bone, bone plate erosion, and joint-space narrowing were scored according to the continuous Ghent University Score System (GUSS ${ }^{\mathrm{TM}}$ ).

Results The disease appeared to be active since $40.0 \%$ and $26,7 \%$ of patients out of the placebo and adalimumab group, respectively, showed at least one new interphalangeal (IP) joint that became erosive during the 12 months follow-up. These differences were not significant and the overall results showed no effect of adalimumab.

Risk factors for progression were then identified and the presence of palpable soft tissue swelling at baseline was recognized as the strongest predictor for erosive progression. In this subpopulation at risk, statistically significant less erosive evolution on the radiological image $(3.7 \%)$ was seen in the adalimumab treated group compared to the placebo group (14.5\%) $(\mathrm{P}=$ 0.009). GUSSTM scoring confirmed a less rapid rate of mean increase in the erosion scores during the first 6 months of treatment in patients in adalimumab-treated patients.

Conclusion Palpable soft tissue swelling in IP joints in patients with erosive HOA is a strong predictor for erosive progression. In these joints adalimumab significantly halted the progression of joint damage compared to placebo.

Moderate to severe hand osteoarthritis is estimated to occur in $5-8 \%$ Caucasian adults above the age of 60 years. ${ }^{12}$ Similar data have been reported in the USA. ${ }^{3}$ In this population, marked destructive changes $^{4-7}$ occur mostly in the distal and proximal interphalangeal joints, which eventually result in considerable disability. ${ }^{89}$

As there is still lack of agreement concerning the nature and specificity of erosive osteoarthritis as a distinct subset of hand osteoarthritis, clear epidemiological data are scarce. In a survey on the entire health district in the Venetian area, radiographic erosive osteoarthritis of the interphalangeal joints occurred in $8.5 \%$ of subjects above the age of 40 years. ${ }^{10}$ These figures were confirmed in two large population studies in which the prevalence of radiographic erosive interphalangeal osteoarthritis in subjects over 55 years of age ranged between $5.0 \%$ and $9.9 \% .{ }^{11} 12$

The changes in both the joint space and subchondral bone that characterise the erosive phase of the interphalangeal finger joints strongly suggest the involvement of pro-inflammatory cytokine cascades known to cause cartilage degradation and bone resorption. Among these, tumour necrosis ractor alpha (TNF $\alpha$ ) directly stimulates osteoclast progenitors of the monocyte/macrophage lineage into osteoclasts, enhances the production of a series of proinflammatory cytokines, eg, interleukin-1 (IL-1), receptor activator for nuclear factor $\kappa \mathrm{B}$ ligand, and increases the rate of tissue remodelling by matrixdegrading proteases. ${ }^{13-15}$

Adalimumab (Humira; Abbott Laboratories, Abbott Park, Illinois) is a bioengineered fully human monoclonal antibody that binds to TNF $\alpha$, preventing it from activating TNF $\alpha$ receptors. ${ }^{16}$ In rheumatoid arthritis (RA), adalimumab slowed down progressive joint destruction. ${ }^{17-19}$ The authors evaluated the efficacy and safety of adalimumab, $40 \mathrm{mg}$ subcutaneous administration, every 2 weeks, to control the structural damage to cartilage and bone, as determined by radiographic assessment, in erosive osteoarthritis of the interphalangeal finger joints in a double blind, placebo controlled randomised clinical trial of 1 year.

\section{PATIENTS AND METHODS \\ Patient inclusion/exclusion criteria}

Sixty patients were recruited from the outpatient rheumatology clinic of the Ghent University Hospital between May 2006 and January 2008. Patients were eligible for study if: (1) they were 18 years or older; (2) had hand osteoarthritis (meeting the American College of Rheumatology criteria) ${ }^{20}$ characterised by painful, inflammatory episodes of the interphalangeal joints; (3) presented with at least one 
interphalangeal finger joint in the ' $E$ ' phase as defined by Verbruggen and Veys ${ }^{7}$ on radiography; and (4) were willing to self-administer subcutaneous injections or allow a suitable person to perform this.

Patients were excluded from the study if they had received previous treatment with any investigational agent within 30 days (or five half lives of the product when longer). Previous treatment with chondroitin sulfate, glucosamine, avocadosoybean unsaponifiables, tetracyclines, corticosteroids or any immunomodulating drug with possible effects on pro-inflammatory cytokine metabolism within 90 days was another reason for exclusion. Patients with chronic inflammatory rheumatic disease (eg, RA, spondylarthropathy, psoriatic arthritis, gout, chondrocalcinosis or other autoimmune diseases) were excluded as well as underlying comorbidities, eg, uncontrolled diabetes, unstable ischaemic heart disease, congestive heart failure, active inflammatory bowel disease, recent stroke (within 3 months before screening), chronic leg ulcer, lymphoproliferative disorders, history of cancer, active hepatitis B or C infection, positive HIV status and persistent or recurrent infections requiring treatment with antibiotics within 30 days before enrolment.

Levels of rheumatoid factor, anti-citrullinated cyclic peptide, erythrocyte sedimentation rate and C-reactive protein were measured at screening. Screening for tuberculosis was done by purified protein derivate skin test and $\mathrm{x}$-ray of the chest.

This investigator-initiated study was declared at www. ClinicalTrials.gov (EudraCT number 2006-000925-71) and complied with the principles of the Declaration of Helsinki. The study protocol was approved by the Ghent University Hospital Ethics Committee. All patients provided written informed consent.

\section{Expected intergroup difference and sample size calculation}

The sample size was calculated based on the results from previous studies ${ }^{7} 2122$ and is applicable for the evolution between baseline and 1 year. The number of patients required for this study was 20 in both treatment arms. It can be estimated that after 1 year, $60 \%$ of patients in the placebo group and $10 \%$ of patients in the adalimumab group will have started the erosive phase in their ' $S$ ' or ' $J$ ' joints. Taking into account 20 patients in each subgroup, a power of $80 \%$ and an $\alpha$-level of $5 \%$, the present study should be able to detect a significant difference between the placebo and treatment groups when $50-55 \%$ of the treated patients have started the erosive phase in one or more non-erosive interphalangeal joints. Considering a drop-out rate comparable with the previous studies ${ }^{22}$ a total of 60 patients were included.

\section{Randomisation and treatment}

Patients received $40 \mathrm{mg}$ adalimumab or placebo administered subcutaneously every 2 weeks for 52 weeks. Randomisation of the study medication was done in by the subsidising company and successive treatment allocation numbers were administered by the investigators following the order of inclusion.

Stable doses of analgesics and anti-inflammatory drugs were allowed as adjuvant therapy. In the case of latent tuberculosis or the presence of other risk factors for activation of latent tuberculosis, the patient was treated with tuberculostatic therapy (isoniazid treatment) at least 3 months before initiation of the trial and continued up to 9 months.

\section{Outcomes}

Primary endpoints were the control of structural damage on radiography. Therefore, posteroanterior radiographs of the
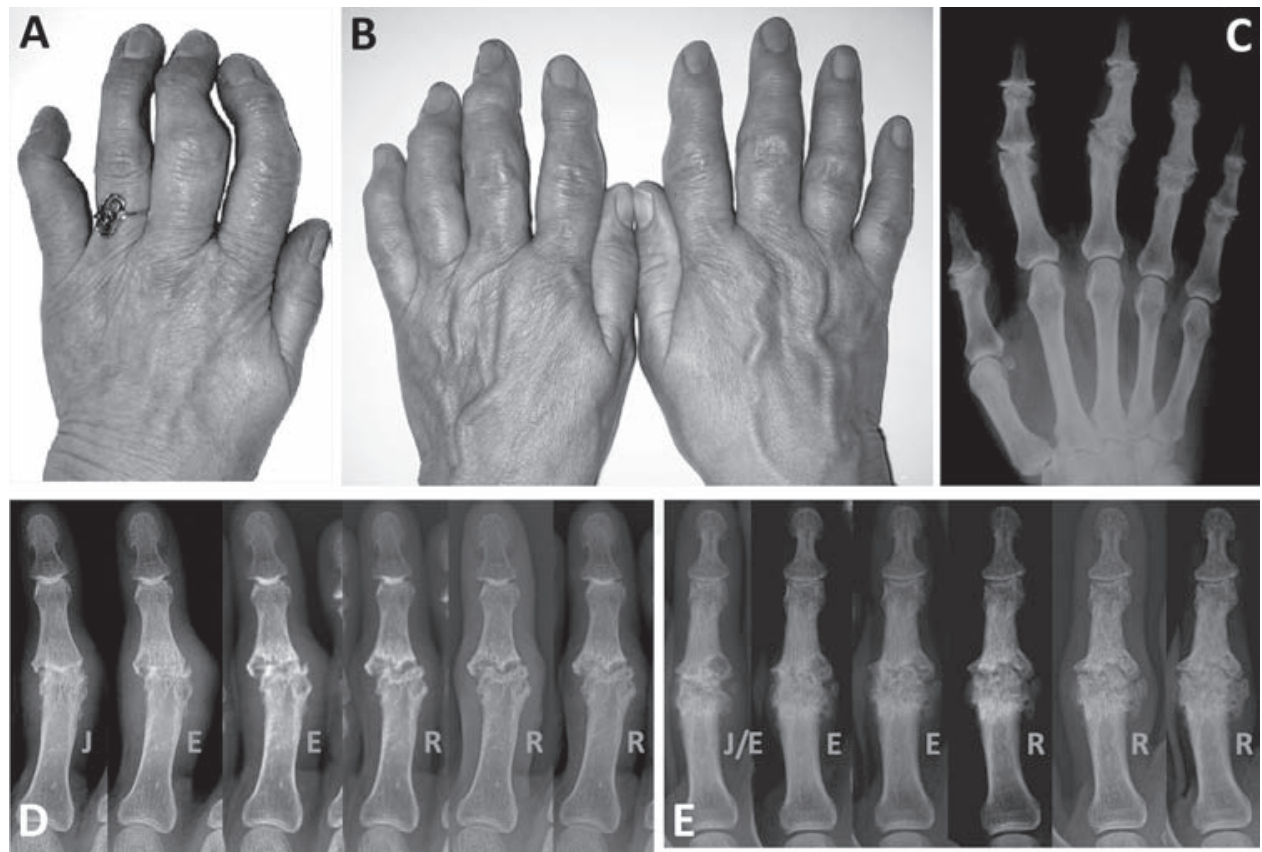

Figure 1 Clinical appearance of erosive osteoarthritis of the interphalangeal finger joints is illustrated in $(A)$ where erosive progression and subsequent remodelling caused functional inability of the second, third and fifth digit, and in (B) where almost all interphalangeal joints of both hands were affected and disabled. Remodelling of the interphalangeal joints in (B) after the erosive process is shown on the corresponding radiograph (C). Typically, metacarpophalangeal joints are spared in this condition. ( $D$ and $E$ ) Erosive progression and subsequent remodelling in two different proximal interphalangeal (PIP) joints is shown in a series of radiographic images taken at 6-month intervals. PIP joints in which the synovial joint space has disappeared ('J' phase) pass through the erosive'E' phase and eventually remodel ('R'). 
hands were obtained at baseline and after 26 and 52 weeks. All interphalangeal joints (distal interphalangeal 2-5 and proximal interphalangeal 2-5) of both hands were scored except the interphalangeal joints of the thumb because of decreased visibility and reproducibility to evaluate on posteroanterior views. A total of 960 interphalangeal joints was scored. In order to score the erosive progression of the interphalangeal joints on the radiographs, two validated scoring systems were used as co-primary endpoints. The first scoring system was based on the consecutive anatomical phases recognised during progressive disease in erosive hand osteoarthritis.7 Non-erosive osteoarthritis joints ('S' or stationary osteoarthritis) have been shown to enter the 'J' phase when the joint space disappears, followed by the 'E' phase when manifest erosive changes occur. Next, the affected interphalangeal joint show signs of repair and the ' $R$ ' (remodelling) phase ensues. A fusion ('F'), as an extreme form of joint remodelling, is seen extremely rarely. Characteristic features and changes are illustrated in figure 1. Anatomical phases of all interphalangeal joints were characterised and changes in phase were recorded by two readers. The percentage of absolute agreement between the readers was $93.6 \%(\kappa=0.92)$. Both readers showed intrareader reliabilities with a percentage of absolute agreement of over $95.9 \%$ ( $\kappa=0.95)$. In case of dispute, agreement was sought by discussing the radiographs. The primary endpoint was a reduction in the numbers of ' $N$ ', 'S' or 'J' joints that become ' $E$ ' within 1 year at the joint and at the patient level.

The second scoring system was the quantitative Ghent University score system (GUSS). ${ }^{23}$ Erosive progression and signs of repair or remodelling were scored by indicating the proportion of normal subchondral bone, subchondral plate and joint space on a 11-point rating scale (range 0-100 with intervals of 10 units). The three scores were summed to obtain the total GUSS score that ranged from 0 (severe joint destruction) to 300 (no damage) for each interphalangeal finger joint. 'E' phase interphalangeal joints thus have, on average, lower GUSS scores than 'J' or ' $R$ ' joints.

Two readers (RW, GV) scored the films independently blinded for order, treatment assignment and clinical response. Interreader and intrareader intraclass coefficients of correlation scores rated excellent for the total GUSS scores: 0.86 and 0.97 , respectively. The smallest detectable change, ${ }^{24}$ as calculated on the mean scores of the two readers was reduced to 18 units after training during routine in-house training sessions. The mean scores of the two readers were used for the GUSS readings.

Secondary endpoints consisted of effects on clinical variables. Clinical assessment was performed by the same experienced examiner, blinded for treatment assignment, at screening, baseline and after $6,12,26,39$ and 52 weeks. This included the evaluation of pain on palpation and the search for palpable swelling as a result of synovial thickening and intra-articular effusion ${ }^{25}$ in 16 interphalangeal finger joints. The grip strength of both hands was measured using a hand grip dynamometer (My-Gripper; Yamasa, Tokei, Japan). The maximum of three attempts was recorded for the dominant and non-dominant hand. Level of pain, morning stiffness and function was registered by the self-reported AUSCAN questionnaire. ${ }^{26}$ The occurrence of any adverse events was questioned at every visit. Blood samples were obtained at baseline and at 6 and 12 months for routine safety tests.

Table 1 Baseline demographic, clinical and radiology data $(N=60)$

\begin{tabular}{|c|c|c|c|c|}
\hline & & $\begin{array}{l}\text { Adalimumab } \\
(\mathrm{N}=\mathbf{3 0})\end{array}$ & $\begin{array}{l}\text { Placebo } \\
(\mathrm{N}=30)\end{array}$ & p Value \\
\hline \multicolumn{5}{|l|}{ Demographics } \\
\hline Women (\%) & & 86.7 & 83.3 & 0.704 \\
\hline $\mathrm{BMI}\left(\mathrm{kg} / \mathrm{m}^{2}\right)$ & $\pm S D$ & $24.8 \pm 3.6$ & $26.5 \pm 4.4$ & 0.122 \\
\hline Age at baseline (years) & $\pm \mathrm{SD}$ & $61.9 \pm 6.1$ & $60.7 \pm 6.9$ & 0.769 \\
\hline Disease duration (years) & $\pm S D$ & $9.6 \pm 6.1$ & $14.4 \pm 8.8$ & 0.016 \\
\hline \multicolumn{5}{|l|}{ Clinical data } \\
\hline AUSCAN pain & $\pm S D$ & $20.4 \pm 9.0$ & $25.1 \pm 11.1$ & 0.008 \\
\hline AUSCAN stiffness & $\pm \mathrm{SD}$ & $5.1 \pm 2.3$ & $5.5 \pm 2.6$ & 0.322 \\
\hline AUSCAN function & $\pm S D$ & $48.4 \pm 20.2$ & $54.1 \pm 21.0$ & 0.006 \\
\hline No of tender joints* & $\pm \mathrm{SD}$ & $3.3 \pm 2.7$ & $5.0 \pm 3.7$ & 0.014 \\
\hline No of palpable effusions* & $\pm S D$ & $3.2 \pm 3.0$ & $2.5 \pm 2.5$ & 0.201 \\
\hline \multicolumn{5}{|l|}{ Maximal grip strength } \\
\hline Dominant hand $(\mathrm{kg})$ & $\pm \mathrm{SD}$ & $18.4 \pm 9.6$ & $19.4 \pm 10.1$ & 0.398 \\
\hline Non-dominant hand (kg) & $\pm \mathrm{SD}$ & $17.2 \pm 7.4$ & $18.3 \pm 9.4$ & 0.258 \\
\hline Analgesics and NSAID intake (N) (\%) & & $9.0(30.0 \%)$ & $8.0(26.7 \%)$ & 0.104 \\
\hline \multicolumn{5}{|l|}{ Radiological data } \\
\hline \multicolumn{5}{|l|}{ Phases at baselinet ( $N=960)$} \\
\hline 'N'/'S' (\%) (N) & & $81.7 \%(392 / 480)$ & $83.3 \%(400 / 480)$ & 0.201 \\
\hline 'J' (\%) (N) & & $5.6 \%(27 / 480)$ & $6.0 \%(29 / 480)$ & 0.658 \\
\hline ‘E' (\%) (N) & & $7.7 \%(37 / 480)$ & $6.4 \%(31 / 480)$ & 0.456 \\
\hline ‘R' (\%) (N) & & $5.0 \%(24 / 480)$ & $4.0 \%(19 / 480)$ & 0.587 \\
\hline 'F' (\%) (N) & & $0.0 \%(0 / 480)$ & $0.2 \%(1 / 480)$ & 0.920 \\
\hline \multicolumn{5}{|l|}{ Thumb base osteoarthritis $\ddagger$} \\
\hline None (N) & & 6 & 7 & 0.902 \\
\hline Mild (N) & & 13 & 11 & 0.811 \\
\hline Moderate/severe (N) & & 11 & 12 & 0.935 \\
\hline
\end{tabular}




\section{Statistical analysis}

Descriptive analyses were performed by the calculation of means, mean differences and proportions. $t$ Tests and $\chi^{2}$ tests were used to explore differences between groups for continuous and dichotomous variables, respectively. For the evaluation of joints, the classic methods neglect the fact that treatment is given on a patient level. Therefore, additional generalised estimating equation (GEE) modelling with a logit link function was used to calculate $\mathrm{OR}$ and differences of means. An additional level (time) was added to the GEE models for longitudinal analysis.

A multi-step method was used to evaluate subgroups of patients that might benefit from anti-TNF $\alpha$ therapy: (1) potential predictors of erosive progression were evaluated by univariate analysis; (2) variables that showed a $50 \%$ difference in odds $(\mathrm{OR}>1.5$ or $\mathrm{OR}<0.667)$ were added in one multivariate GEE model; (3) significant predictors from that model were evaluated by interaction terms with therapy (placebo or adalimumab) in further regression analyses. For these subgroup analyses, disease progression was assessed using the anatomical phase scoring system ${ }^{7}$ and the GUSS ${ }^{23}$ as follows: for the evaluation of classic calculated $\mathrm{OR}$, a new erosive joint was defined if a joint with a baseline ' $N$ ', 'S' or 'J' phase evolved to an 'E' phase after 1 year. In the GEE models, 'E' joints were compared with all other phases at the two different time points. For the evaluation of the GUSS, differences between baseline were calculated and the different scores of these joints showing no evolution were imputed with zero.

Concerning the descriptive analyses and the analysis of differences, the last observation carried forward method was used in the case of missing values (one patient (placebo) missed the 12 -month visit). For the calculation of inference by GEE modelling, no imputation was used. All statistics were calculated using SPSS 16.

\section{RESULTS \\ Patient characteristics}

Sixty patients were enrolled in the study. Both adalimumab and placebo-treated groups included 30 subjects. None of the patients were rheumatoid factor or anti-citrullinated cyclic peptide positive. Six patients, of which four were in the adalimumab group, showed positive purified protein derivate skin tests with no signs of active pulmonary tuberculosis. Here, izoniazid treatment was initiated. Demographic characteristics between both treatment groups were compared (table 1). A flow diagram of the study is provided in figure 2 .

\section{Structural changes in interphalangeal joints: overall treatment effects}

Anatomical phases and changes over time of all 960 interphalangeal joints were characterised.

At baseline, 68 out of 960 (7.1\%) interphalangeal finger joints were in the ' $E$ ' phase, $43(4.5 \%)$ in the 'R' phase and one joint was fused. Of the remaining 848 (88.3\%) interphalangeal finger joints in the 'N', 'S' or 'J' phases at baseline, 24 (2.8\%) from 20 patients became fully erosive interphalangeal joints after 1 year.

Fifteen out of 429 (3.6\%) 'N', 'S' or 'J' joints from placebotreated patients showed an evolution to an ' $\mathrm{E}$ ' joint compared with nine of $419(2.1 \%)$ joints in adalimumab-treated patients (GEE OR 1.43; 95\% CI 0.65 to 3.16, $\mathrm{p}=0.37$ ). Active disease, defined by the presence of at least one new erosive joint over 12 months, was present in 12 of $30(40.0 \%)$ and eight of $30(26.7 \%)$

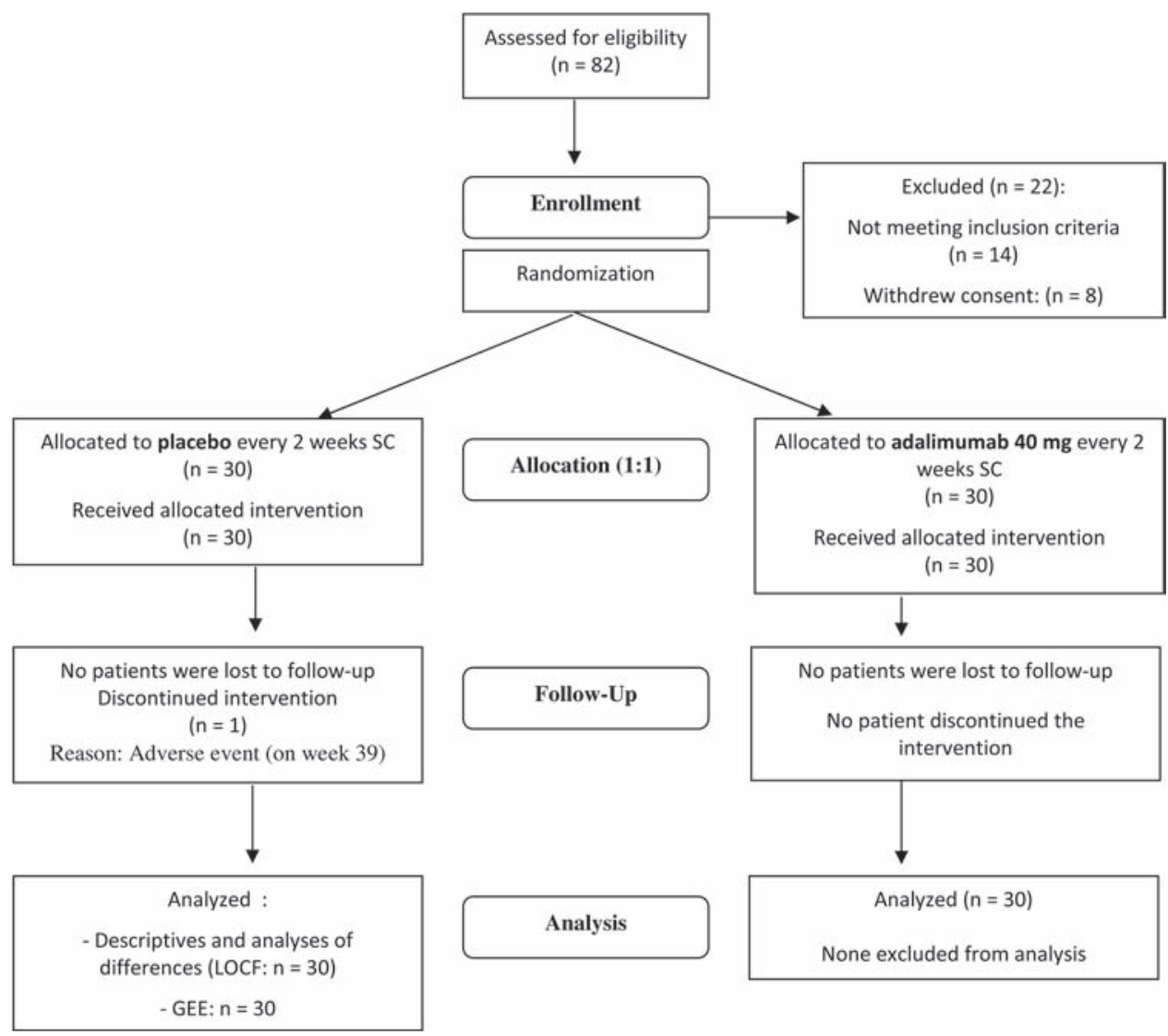

Figure 2 Flow diagram of the study. GEE, general estimating equation; LOCF, last observation carried forward; SC, subcutaneous. 


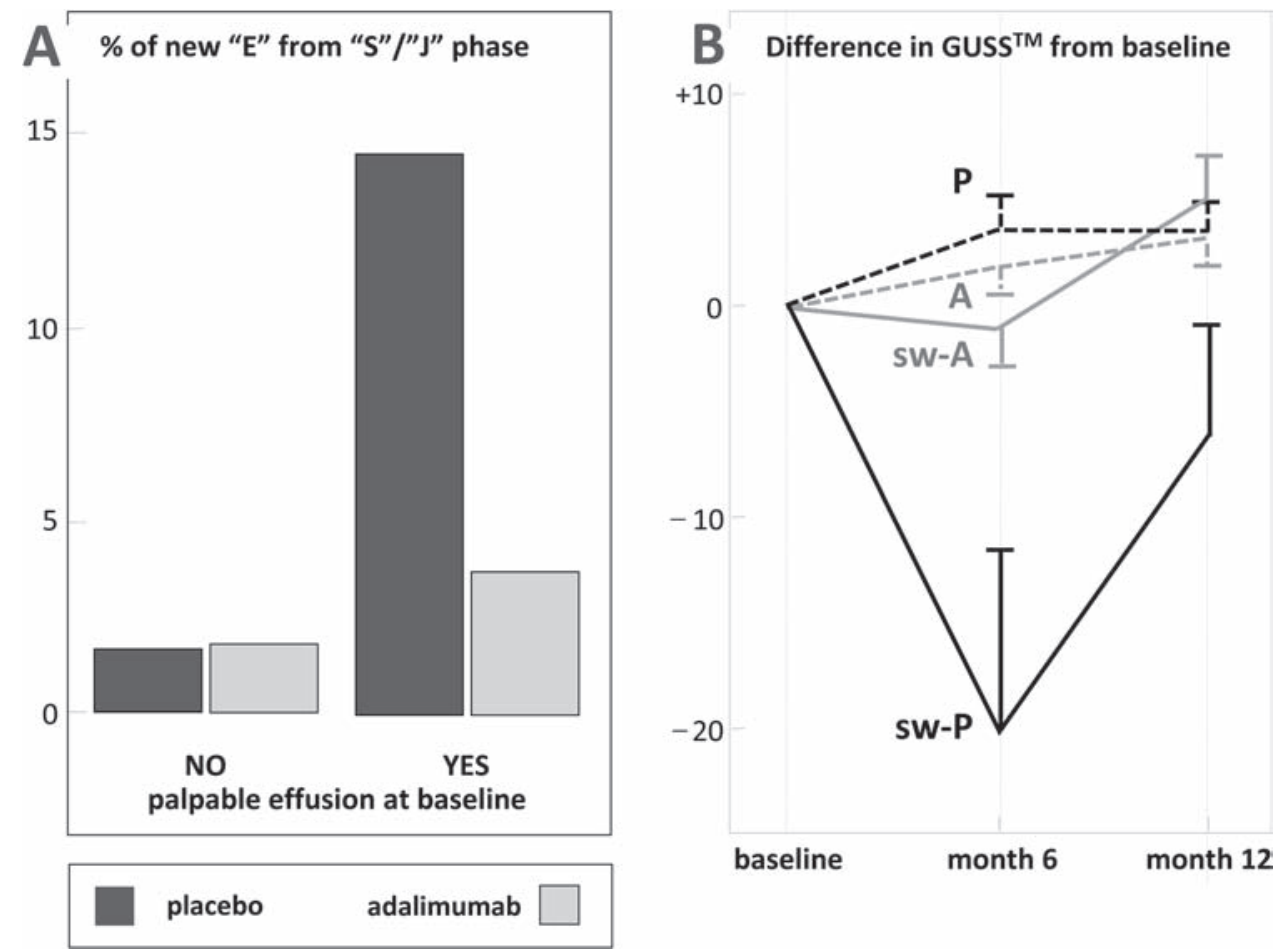

Figure 3 (A) Effect of adalimumab in interphalangeal finger joints at high risk of erosive disease. Tenfold increase in percentage numbers of interphalangeal finger joints that progress to erosive disease, as observed in joints showing palpable effusion at baseline, are significantly reduced in the treatment group. (B) Ghent University score system (GUSS) scores in interphalangeal finger joints. Differences in GUSS scores between baseline (normalised), 6 and 12 months in interphalangeal joints with and without palpable effusion in treatment and placebo groups. Mean values and SEM are given. P, Non-swollen interphalangeal—placebo; A, non-swollen interphalangeal—adalimumab; sw-P, swollen interphalangeal—placebo; sw-A, swollen interphalangeal—adalimumab.

Table 2 Exploration of potential risk factors for more erosive disease

\begin{tabular}{|c|c|c|c|}
\hline Continuous variables & Mean difference $(\mathrm{SE})^{\dagger}$ & GEE OR (95\% CI) & p Value ${ }^{\ddagger}$ \\
\hline Disease duration & $+4.20(1.60)$ & 0.95 (0.91 to 0.98$)$ & 0.014 \\
\hline Age & $+1.90(1.80)$ & 1.01 (0.96 to 1.08$)$ & 0.560 \\
\hline Baseline CRP & $-0.02(0.05)$ & 0.99 (0.95 to 1.05$)$ & 0.970 \\
\hline Baseline ESR & +1.98 (2.10) & $1.00(0.99$ to 1.00$)$ & 0.440 \\
\hline Dichotomous variables & $\mathrm{OR}^{*}(95 \% \mathrm{Cl})$ & GEE OR (95\% Cl) & p Value ${ }^{\ddagger}$ \\
\hline Tender joints at baseline (N) & $3.30(1.40$ to 7.60$)$ & $2.50(1.10$ to 5.70$)$ & 0.030 \\
\hline Palpable effusion at baseline & $5.30(2.30$ to 12.0$)$ & $4.40(2.10$ to 8.80$)$ & 0.001 \\
\hline PIP joint vs DIP joint & $1.50(0.60$ to 3.20$)$ & $0.12(0.78$ to 1.03$)$ & 0.790 \\
\hline
\end{tabular}

patients in the placebo and adalimumab-treated groups, respectively ( $\mathrm{p}=0.09)$. The differences were not significant.

\section{Structural changes in interphalangeal joints: predictors for erosive evolution}

From the demographic data, three variables were recognised as potential predictors for erosive evolution: disease duration, the number of tender joints at baseline and the number of joints with palpable effusion at baseline. The latter variable was associated with the highest risk of erosive evolution (table 2).

\section{Treatment effects on interphalangeal finger joints at risk of erosive evolution}

GEE modelling (with a logit link function) was used to explore the interaction terms between treatment and each selected risk factor and revealed a significant interaction term for palpable effusion with therapy.

Adalimumab treatment prevents erosive evolution in patients with soft tissue swelling at baseline: nine of these inflamed interphalangeal joints out of $62(14.5 \%)$ at baseline became erosive under placebo treatment versus three out of $81(3.7 \%)$ under adalimumab therapy (GEE OR 4.57; 95\% CI 1.46 to 14.3 , $p=0.009$; figure $3 \mathrm{~A}$ ). Similarly, treatment with adalimumab causes a fourfold reduction in the risk of erosive progression compared with placebo.

In addition, we evaluated whether the differences between groups, observed with the anatomical phase scoring method, could be confirmed by GUSS.

Joints showing baseline palpable swelling from patients treated with placebo showed significantly more change of GUSS scores towards progression between baseline and 6 months than 
Table 3 Longitudinal clinical data

\begin{tabular}{llll}
\hline Variable & Adalimumab $\mathbf{N}=\mathbf{3 0})$ & Placebo $(\mathbf{N}=\mathbf{3 0})$ & p Value \\
\hline AUSCAN pain & $5.4(9.8)$ & $1.7(13.1)$ & 0.063 \\
AUSCAN stiffness & $0.4(2.1)$ & $0.1(3.0)$ & 0.721 \\
AUSCAN function & $1.2(18.5)$ & $2.0(17.9)$ & 0.133 \\
No of tender joints & $0.7(2.3)$ & $1.4(4.8)$ & 0.238 \\
No of joints with palpable effusion & & & \\
Maximal grip strength (kg) & $1.7(2.7)$ & $1.0(2.4)$ & 0.814 \\
Dominant hand & $0.8(1.2)$ & $1.2(1.8)$ & 0.231 \\
Maximal grip strength & & $0.2(1.1)$ & 0.281 \\
Non-dominant hand & $0.9(1.3)$ & &
\end{tabular}

Data shown are mean differences (SD) in changes of scores from baseline to week 52 .

Safety results are reported in table 4. More adverse events were reported in the adalimumab group $(\mathrm{N}=13)$ than in the placebo group $(N=8)$, although more infectious adverse events were seen in the placebo group (four vs only two in the adalimumab group). Three infections required antibiotics. All adverse events were graded as mild to moderate in severity and only one case required withdrawal from the study. This patient withdrew consent after experiencing vertigo and hypertension 9 months after baseline. This patient was treated with placebo. No serious adverse events or malignancies occurred. Biological routine safety blood tests revealed no problems.

Table 4 Adverse events

\begin{tabular}{lll}
\hline & Placebo $(\mathbf{N}=\mathbf{3 0})$ & Adalimumab $\mathbf{( N = 3 0 )}$ \\
\hline Adverse events & 8 & 13 \\
Patients experiencing at least one & 7 & 7 \\
adverse event 8 & & \\
Infections & 4 & 2 \\
Upper respiratory tract* & 1 & 2 \\
Urinary tract* & 1 & 0 \\
Folliculitis* $^{*}$ & 1 & 0 \\
Conjunctivitis* & 1 & 0 \\
Non-infectious events & 4 & 11 \\
Injection site reactions* & 0 & 1 \\
Weight loss & 0 & 1 \\
Weight gain & 1 & 0 \\
Vertigo & 1 & 2 \\
Angor pectoris & 1 & 0 \\
Urticaria & 0 & 1 \\
Headache & 0 & 1 \\
Pyrosis (heartburn) & 0 & 1 \\
Tendinitis & 0 & 1 \\
Hypertension & 0 & 1 \\
Erythematous cutaneous rash & 0 & 1 \\
Toxicoderma & 0 & 1 \\
Shoulder pain & 1 & 0 \\
\hline
\end{tabular}

No serious adverse events or malignancies were reported; no significant differences in numbers of adverse events; adverse events marked with * may be treatment related.

from patients treated with adalimumab (mean difference -20.0 , $\mathrm{SE}$ 9.9, $\mathrm{p}=0.022$; GEE model; figure 3B). These interphalangeal joints started to repair and remodel during further follow-up (figure 1D,E). Renewal of the tissue structures then resulted in higher GUSS scores after 12 months. GUSS scores remained stable under adalimumab in joints showing baseline palpable swelling. Non-swollen interphalangeal joints did not show any change in their GUSS scores.

\section{Clinical variables}

Differences in clinical variables between baseline and week 52 are shown in table 3. No significant changes were observed between both treatment groups after 1 year (table 4).

\section{DISCUSSION}

Erosive hand osteoarthritis targets interphalangeal joints and shows severe radiographic subchondral bone destruction and cartilage attrition with poor long-term functional outcome, ${ }^{8} 9$ 27-29 and functional impairment caused by hand osteoarthritis was reported to be similar in severity to that in RA. ${ }^{30}$ Meaningful treatment should deal with the destructive events during the disease in order to prevent the progression of structural remodelling. The destructive events, ie, the osteolytic processes and the disappearance of the articular cartilage in osteoarthritis, are most likely mediated by the same cytokine cascades operational in other inflammatory rheumatic conditions, eg, RA and psoriatic arthritis. ${ }^{31-35}$ Here in states of skeletal inflammation TNF $\alpha$ is produced, which is the dominant cytokine present in inflammation-associated joint tissue destruction. TNF $\alpha$ directly stimulates osteoclast progenitors of the monocyte/macrophage lineage to differentiate to osteoclasts, ${ }^{13} 14$ and augments receptor activator for nuclear factor $\kappa \mathrm{B}$ ligand expression, ${ }^{14} \mathrm{IL}-1$ and its signalling receptor IL-1R1 messenger RNA ${ }^{14} 15$ in bone marrow stromal cells and synovial fibroblasts. It is likely that TNF $\alpha$ activity may be responsible for the destructive processes observed in cartilage and subchondral bone in erosive interphalangeal joint osteoarthritis

TNF $\alpha$ blockade is thus a possible therapeutic option in erosive interphalangeal joint osteoarthritis as it could help to preserve tissue structure and hand function as a whole.

For this study we selected patients showing active erosive osteoarthritis in one or more of their interphalangeal finger joints. The natural history in these patients showed high rates of progression, as almost half of them on placebo had one or more non-erosive interphalangeal joints at baseline that became erosive during follow-up.

Fewer adalimumab-treated patients developed erosive osteoarthritis in their interphalangeal joints: $26.7 \%$ of patients in $2.1 \%$ of their non-erosive interphalangeal joints, whereas $40.0 \%$ of the placebo-treated patients did so in $3.6 \%$ of their non-erosive finger joints. These differences were not significant and the overall results thus showed no therapeutic effect of adalimumab.

However, the data obtained in the placebo group allowed potential predictors for erosive evolution in interphalangeal finger joint osteoarthritis to be identified. Most significantly, soft tissue swelling at study entry was a key predictor for erosive evolution. In the placebo group, a 10-fold increase in progression to the erosive ' $E$ ' phase occurred in non-erosive interphalangeal joints presenting with soft tissue swelling. Neutralising TNF $\alpha$ significantly slowed down the progression of structural damage in these inflamed interphalangeal joints.

These results were confirmed when erosive progression in this particular form of hand osteoarthritis was scored by the novel scoring system GUSS, designed to monitor disease progression within a shorter time. Anti-TNF $\alpha$ therapy prevented GUSS from changing in interphalangeal joints with palpable effusion at baseline. In the placebo group, GUSS scores dropped dramatically in 
the interphalangeal joints before increasing towards the end of the follow-up period as a result of spontaneous repair. ${ }^{7}$ It can be presumed that remodelling of an affected interphalangeal joint after major tissue damage will result in a marked change of the original anatomy and loss of the normal function.

Anti-TNF therapy retarded erosive progression in RA and psoriatic arthritis, ${ }^{17-19}$ and clinical improvement was associated with the inhibition of TNF activity in most although not in all of these studies. ${ }^{17}{ }^{19}$ In fact, erosive progression could be halted in RA during anti-TNF therapy even though clinical signs of joint inflammation remained. ${ }^{36}$

Also in our study in hand osteoarthritis, anti-TNF treatment did not show any effect on inflammation, as assessed by the number of joints with palpable swelling that remained constant throughout follow-up. The direct causal relationship between synovitis and bone erosions in RA has been questioned, and erosions and the signs of synovitis may represent different pathological processes. ${ }^{37}$

Thus far, two open-label studies have reported on the effects of TNF inhibition on clinical variables in hand osteoarthritis. ${ }^{38}$ 39 One underpowered open-label study failed to show any clinical effect, ${ }^{38}$ and it has to be admitted that our study may also have been underpowered to perceive clinical change as it was powered to detect structure modification. Another singleblinded study on 10 patients in which 56 and 34 interphalangeal joints were injected with infliximab and placebo, respectively, showed a significant reduction of spontaneous pain and pain on pressure when individual joints were studied. ${ }^{39}$ Although conclusions are hard to take from open-label studies, it may point to a weakness in our study in that we used the AUSCAN, which was designed to measure overall hand function. TNF $\alpha$ blockade, when retarding disease progression in some selected target interphalangeal joints, was not supposed to alter residual hand function and pain caused by multiple deformities that previously occurred in other finger joints. It may be expected, however, that treatment interventions given at appropriate time intervals will help to preserve tissue structure and hand function as a whole over time.

The tolerability and safety profiles of adalimumab in our patients with erosive hand osteoarthritis were as good as those in patients with other systemic rheumatic diseases. ${ }^{17,40}$

This placebo controlled randomised clinical trial offers the first proof of principle that TNF $\alpha$ neutralisation by adalimumab is able to slow down the progression of structural damage in erosive interphalangeal finger joint osteoarthritis. This effect was only seen in interphalangeal joints with destructive features on radiography and with palpable effusion. Larger phase III studies should be performed to confirm these results.

Acknowledgments The study was funded by an educational grant from Abbott Laboratories, USA. RW is a research fellow supported by a Ghent University coordinated research initiative (GOA) grant (BOF07/GOA/002). BVC is a postdoctoral researcher of the Research Foundation Flanders (FWO).

Ethics approval The study protocol was approved by the Ghent University Hospital Ethics Committee.

Patient consent Obtained.

Competing interests None.

Provenance and peer review Not commissioned; externally peer reviewed

\section{REFERENCES}

1. Mannoni A, Briganti MP, Di Bari M, et al. Epidemiological profile of symptomatic osteoarthritis in older adults: a population based study in Dicomano, Italy. Ann Rheum Dis 2003;62:576-8.

2. Bagge $\mathbf{E}$, Bjelle A, Valkenburg HA, et al. Prevalence of radiographic osteoarthritis in two elderly European populations. Rheumatol Int 1992;12:33-8.
3. Dillon CF, Hirsch R, Rasch EK, et al. Symptomatic hand osteoarthritis in the United States: prevalence and functional impairment estimates from the third U.S. National Health and Nutrition Examination Survey, 1991-1994. Am J Phys Med Rehabil 2007;86:12-21.

4. Stecher RM, Hauser H. Heberden's nodes; the roentgenological and clinical appearance of degenerative joint disease of the fingers. Am J Roentgenol Radium Ther 1948;59:326-37.

5. Crain DC. Interphalangeal osteoarthritis. JAMA 1961;175:1049-53.

6. Peter JB, Pearson CM, Marmor L. Erosive osteoarthritis of the hands. Arthritis Rheum 1966:9:365-88.

7. Verbruggen G, Veys EM. Numerical scoring systems for the anatomic evolution of osteoarthritis of the finger joints. Arthritis Rheum 1996;39:308-20.

8. Zhang $\mathbf{Y}$, Niu J, Kelly-Hayes M, et al. Prevalence of symptomatic hand osteoarthritis and its impact on functional status among the elderly: the Framingham Study. Am J Epidemiol 2002;156:1021-7.

9. Dahaghin S, Bierma-Zeinstra SM, Ginai AZ, et al. Prevalence and pattern of radiographic hand osteoarthritis and association with pain and disability (the Rotterdam study). Ann Rheum Dis 2005;64:682-7.

10. Cavasin F, Punzi L, Ramonda R, et al. [Prevalence of erosive osteoarthritis of the hand in a population from Venetian area]. Reumatismo 2004;56:46-50.

11. Kwok WY, Kloppenburg M, Rosendaal FR, et al. Erosive hand osteoarthritis: its prevalence and clinical impact in the general population and symptomatic hand osteoarthritis. Ann Rheum Dis 2011;70:1238-42.

12. Haugen IK, Englund M, Aliabadi P, et al. Prevalence, incidence and progression of hand osteoarthritis in the general population: the Framingham Osteoarthritis Study. Ann Rheum Dis 2011;70:1581-6.

13. Kobayashi K, Takahashi N, Jimi E, et al. Tumor necrosis factor alpha stimulates osteoclast differentiation by a mechanism independent of the ODF/RANKL-RANK interaction. J Exp Med 2000;191:275-86.

14. Komine M, Kukita A, Kukita T, et al. Tumor necrosis factor-alpha cooperates with receptor activator of nuclear factor kappaB ligand in generation of osteoclasts in stromal cell-depleted rat bone marrow cell culture. Bone 2001;28:474-83.

15. Wei S, Kitaura H, Zhou P, et al. IL-1 mediates TNF-induced osteoclastogenesis. $J$ Clin Invest 2005;115:282-90.

16. Arora T, Padaki R, Liu L, et al. Differences in binding and effector functions between classes of TNF antagonists. Cytokine 2009;45:124-31.

17. Keystone EC, Kavanaugh AF, Sharp JT, et al. Radiographic, clinical, and functional outcomes of treatment with adalimumab (a human anti-tumor necrosis factor monoclonal antibody) in patients with active rheumatoid arthritis receiving concomitant methotrexate therapy: a randomized, placebo-controlled, 52-week trial. Arthritis Rheum 2004;50:1400-11.

18. Hoff M, Kvien TK, Kälvesten J, et al. Adalimumab therapy reduces hand bone loss in early rheumatoid arthritis: explorative analyses from the PREMIER study. Ann Rheum Dis 2009;68:1171-6.

19. Møller Døhn U, Boonen A, Hetland ML, et al. Erosive progression is minimal, but erosion healing rare, in patients with rheumatoid arthritis treated with adalimumab. A 1 year investigator-initiated follow-up study using high-resolution computed tomography as the primary outcome measure. Ann Rheum Dis 2009;68:1585-90.

20. Altman R, Alarcón G, Appelrouth D, et al. The American College of Rheumatology criteria for the classification and reporting of osteoarthritis of the hand. Arthritis Rheum 1990;33:1601-10.

21. Verbruggen G, Goemaere S, Veys EM. Chondroitin sulfate: S/DMOAD (structure/ disease modifying anti-osteoarthritis drug) in the treatment of finger joint $\mathrm{OA}$ Osteoarthritis Cartilage 1998;6 (Suppl A):37-8.

22. Verbruggen G, Goemaere $S$, Veys EM. Systems to assess the progression of finger joint osteoarthritis and the effects of disease modifying osteoarthritis drugs. Clin Rheumatol 2002;21:231-43.

23. Verbruggen G, Wittoek R, Vander Cruyssen B, et al. Morbid anatomy of 'erosive osteoarthritis' of the interphalangeal finger joints: an optimised scoring system to monitor disease progression in affected joints. Ann Rheum Dis 2010;69:862-7.

24. Ravaud P, Giraudeau B, Auleley GR, et al. Assessing smallest detectable change over time in continuous structural outcome measures: application to radiological change in knee osteoarthritis. J Clin Epidemiol 1999;52:1225-30.

25. Wittoek $\mathbf{R}$, Carron P, Verbruggen $G$. Structural and inflammatory sonographic findings in erosive and non-erosive osteoarthritis of the interphalangeal finger joints. Ann Rheum Dis 2010;69:2173-6.

26. Bellamy N, Campbell J, Haraoui B, et al. Dimensionality and clinical importance of pain and disability in hand osteoarthritis: development of the Australian/Canadian (AUSCAN) Osteoarthritis Hand Index. Osteoarthritis Cartilage 2002;10:855-62.

27. Pattrick M, Aldridge $S$, Hamilton E, et al. A controlled study of hand function in nodal and erosive osteoarthritis. Ann Rheum Dis 1989;48:978-82.

28. Kjeken I, Dagfinrud H, Slatkowsky-Christensen B, et al. Activity limitations and participation restrictions in women with hand osteoarthritis: patients' descriptions and associations between dimensions of functioning. Ann Rheum Dis 2005;64:1633-8.

29. Jones G, Cooley HM, Bellamy N. A cross-sectional study of the association between Heberden's nodes, radiographic osteoarthritis of the hands, grip strength, disability and pain. Osteoarthritis Cartilage 2001;9:606-11. 
30. Leeb BF, Sautner J, Andel I, et al. SACRAH: a score for assessment and quantification of chronic rheumatic affections of the hands. Rheumatology (Oxford) 2003:42:1173-8

31. Saklatvala J. Tumour necrosis factor alpha stimulates resorption and inhibits synthesis of proteoglycan in cartilage. Nature 1986;322:547-9.

32. Polzer K, Schett G, Zwerina J. The lonely death: chondrocyte apoptosis in TNFinduced arthritis. Autoimmunity 2007;40:333-6.

33. Fernandes JC, Martel-Pelletier J, Pelletier JP. The role of cytokines in osteoarthritis pathophysiology. Biorheology 2002;39:237-46.

34. Wang J, Verdonk P, Elewaut $\mathrm{D}$, et al. Homeostasis of the extracellular matrix of normal and osteoarthritic human articular cartilage chondrocytes in vitro. Osteoarthritis Cartilage 2003;11:801-9.

35. Romas E, Gillespie MT, Martin TJ. Involvement of receptor activator of NFkappaB ligand and tumor necrosis factor-alpha in bone destruction in rheumatoid arthritis. Bone 2002;30:340-6.
36. Døhn UM, Skjødt H, Hetland ML, et al. No erosive progression revealed by $\mathrm{MRI}$ in rheumatoid arthritis patients treated with etanercept, even in patients with persistent MRI and clinical signs of joint inflammation. Clin Rheumatol 2007;26:1857-61

37. Kirwan JR. The relationship between synovitis and erosions in rheumatoid arthritis. Br J Rheumatol 1997;36:225-8

38. Magnano MD, Chakravarty EF, Broudy C, et al. A pilot study of tumor necrosis factor inhibition in erosive/inflammatory osteoarthritis of the hands. J Rheumatol 2007;34:1323-7.

39. Fioravanti A, Fabbroni M, Cerase A, et al. Treatment of erosive osteoarthritis of the hands by intra-articular infliximab injections: a pilot study. Rheumatol Int 2009;29:961-5.

40. Burmester GR, Mease P, Dijkmans BA, et al. Adalimumab safety and mortality rates from global clinical trials of six immune-mediated inflammatory diseases. Ann Rheum Dis 2009;68:1863-9. 